\title{
NÍVEIS PLASMÁTICOS DE ZINCO E ANTROPOMETRIA DE CRIANÇAS DA PERIFERIA DE CENTRO URBANO NO BRASIL
}

Rosa Maria Duarte Fávaro* Helio Vannucchi*

\begin{abstract}
FÁVARO, R.M.D. \& VANNUCCHI, H. Níveis plasmáticos de zinco e antropometria de crianças da periferia de centro urbano no Brasil. Rev.Saúde públ., S. Paulo, 24: 5 - 10, 1990.
\end{abstract}

RESUMO: Foi determinada a concentração plasmática de zinco em crianças pertencentes a famillias de baixa renda que residiam em três bairros da periferia de Ribeirão Preto, SP (Brasil), e verificada uma possível correlação desses níveis com algumas medidas antropométricas. Foram estudadas 126 crianças de 2 a 7 anos. Os núveis plasmáticos de zinco foram inferiores a $70 \mu \mathrm{g} \% \mathrm{em} 13 \%$ das crianças estudadas. As medidas antropométricas mostraram que $42,9 \%$ das crianças estudadas apresentavam peso, $6,3 \%$ apresentavam altura e $9,5 \%$ apresentavam peso/altura inferiores a $90 \%$ do percentil 50 do National Center for Health Statistics (NCHS). A prega tricipital e a circunferência braqueal e muscular foram inferiores a $90 \%$ do percentil 50 do NCHS em respectivamente $65,8 \%, 11 \%$ e 7,5\% das crianças estudadas. Não se observou correlação significante entre os níveis plasmáticos de zinco e os parâmetros antropométricos estudados.

DESCRITORES: Zinco, sangue. Antropometria. Fatores sócio-econômicos.

\section{INTRODUÇÃO}

O zinco é um nutriente essencial ao organismo humano, sendo constituinte de diversas enzimas envolvidas no metabolismo intermediário ${ }^{24}$.

A deficiência severa de zinco ocorre com maior freqüência em doenças como a cirrose alcoblica e acrodermatite enteropática, sendo que as manifestaçōes clínicas classicamente descritas são: alteraçōes neuropsiquiátricas, dermatite perioral e de extremidades, diarréia e alopecia ${ }^{24}$.

A deficiência leve de zinco pode ocorrer associada à ingestão insuficiente ou à baixa biodisponibilidade desse mineral na dieta, tendo sido observada em comunidades por vários autore $s^{4,6}$, 31, 33. Krebs e col.21, ao estudarem um grupo de crianças deficientes, em Denver, demonstraram um aumento da ingestão de alimentos quando havia suplementaçăo com zinco em comparação às crianças que receberam placebo. A associação de deficiência de zinco com hipogeusia é ainda discutida requerendo observações posteriores ${ }^{1}$. A melhoria da resposta imune com a suplementação de zinco foi descrita em pacientes obesos ${ }^{7}$, em crianças com Síndrome de Down ${ }^{2}$, e em idosos ${ }^{3}$. O hipogonadismo, com desenvolvimento retardado dos caracteres sexuais secundários, foi mostrado em adolescentes com nanismo nutricional e deficiência de zinco no Egito $^{25}$ e Irã ${ }^{16}$. A deficiência leve de zinco tem sido apontada como causa reversível de disfunção gonadal secundária em pacientes com uremia ${ }^{23}$ e também oligospermia ${ }^{1}$. Crianças, com deficiência leve de zinco, quando receberam suplementação desse mineral, apresentaram melhor recuperação do peso comparadas às que receberam placebo ${ }^{31}$. Halsted e col. ${ }^{16}$ demonstraram, ainda, maior ganho na altura por adolescentes suplementados com zinco.

No Brasil, poucos estudos populacionais sobre o estado nutricional relativo ao zinco têm sido publicados, e os existentes mostram baixas concentrações no cabelo ou plasma de fração significativa dos indivíduos estudados, principalmente crianças $^{10,11,12,26}$. Lamounier e col. 2 demonstraram, ainda, que a concentração de zinco no leite de mães de baixo nível sócio-econômico, moradoras do $\mathrm{Mu}$ nicípio de Ribeirão Preto, SP, é inferior às recomendaçð̃es diárias desse mineral para lactentes.

O presente estudo teve por objetivo determinar as concentraçőes plasmáticas de zinco de crianças de baixo nível sócio-econômico, moradoras na periferia de Ribeirão Preto, e correlacioná: los aos parâmetros antropométricos.

\section{MATERIAL E MÉTODOS}

\section{Indivíduos - Casuística}

Foram estudadas 126 crianças, 69 do sexo masculino e 57 do sexo feminino, de 2 a 7 anos de idade, moradoras de três bairros periféricos de Ribeirão Preto. Essas crianças pertenciam a famílias cuja renda mensal era inferior a três salários mínimos. As familias foram selecionadas ao acaso, sendo que participaram do estudo aquelas com crianças

* Departamento de Clínica Médica da Faculdade de Medicina de Ribeirão Preto da Universidade de São Paulo - Av. Bandeirantes, 3.900 - 14049 - Ribeirão Preto, SP — Brasil. 
pré-escolares e cujos pais permitiram a participação no projeto. Das crianças estudadas, $45 \%$ freqüentavam parques infantis próximos de suas residências.

\section{Medidas Antropométricas}

Peso, altura, prega tricipital e circunferência braquial foram medidas usando procedimentos padronizados ${ }^{20}$. Os valores de referência usados para comparar os dados de peso e altura foram os do National Center for Health Statistics (NCHS), reportados por Hamill e col. ${ }^{19}$ Os padrões de referência usados para comparação da prega tricipital, circunferência braquial e circunferência muscular foram também os do NCHS, descritos por Frisancho ${ }^{13}$. Os parâmetros antropométricos das crianças estudadas foram comparados de acordo com sexo e idade, com valores do percentil 50 do NCHS.

\section{Níveis Plasmáticos de Zinco}

O sangue venoso foi coletado em jejum e colocado em tubos de polietileno contendo EDTA como anticoagulante. A seguir, os tubos eram transportados para o laboratório onde o sangue era centrifugado, o plasma separado e então congelados $\left(-15^{\circ} \mathrm{C}\right)$ até o momento das dosagens. $O$ zinco plasmático foi medido por espectrofotometria de ab- sorção atômica, usando-se um aparelho PerkinElmer 290B, após diluição adequada com água deionizada (1:3). Os padröes foram preparados com solução de titrisol-Merck $\left(\mathrm{ZnCl}_{2}\right)$ e com solução de revestimento para análises de soro e plasma (Merck). Todo o material utilizado para a coleta e dosagem das amostras foi lavado com solução de Extran (Merck) e a seguir com solução de ácido nítrico P.A. a $30 \%$ e enxaguado com água deionizada.

Consideraram-se deficientes os níveis plasmáticos de zinco inferiores a $70 \mu \mathrm{g} \% 4,14,17$.

\section{Análise Estatística}

Os valores de zinco plasmático apresentaram uma distribuição normal e os grupos de dados foram comparados por meio de análise de variância $^{29}$. Utilizou-se análise de regressão para determinar os coeficientes de correlação entre níveis plasmáticos de zinco e parâmetros antropométri$\cos ^{29}$.

\section{RESULTADOS}

\section{Parâmetros Antropométricos}

A distribuição das 126 crianças estudadas de
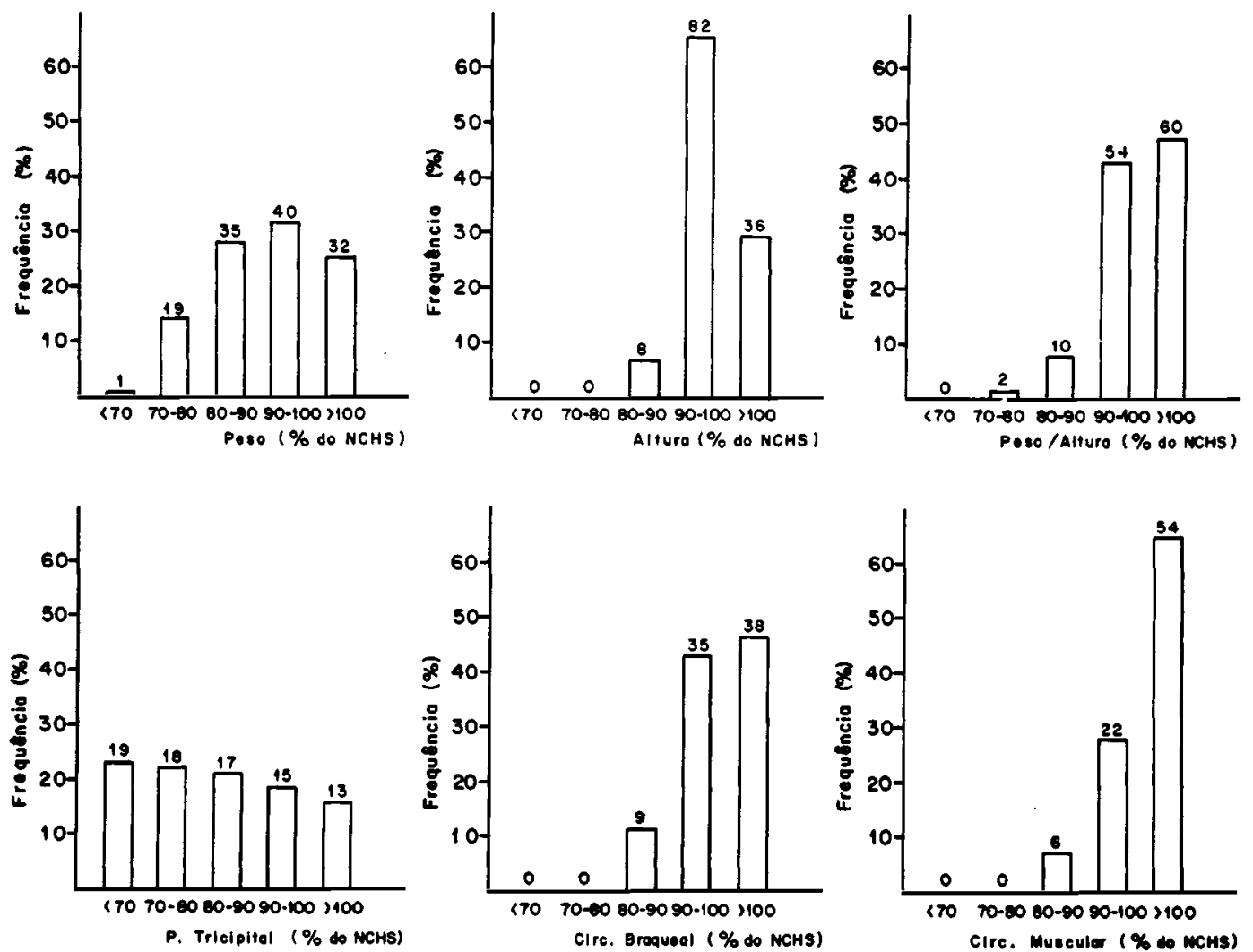

Fig. 1 - Freqüência de alterações dos parâmetros antropométricos de crianças de baixa renda da periferia de Ribeirão Preto, SP. 


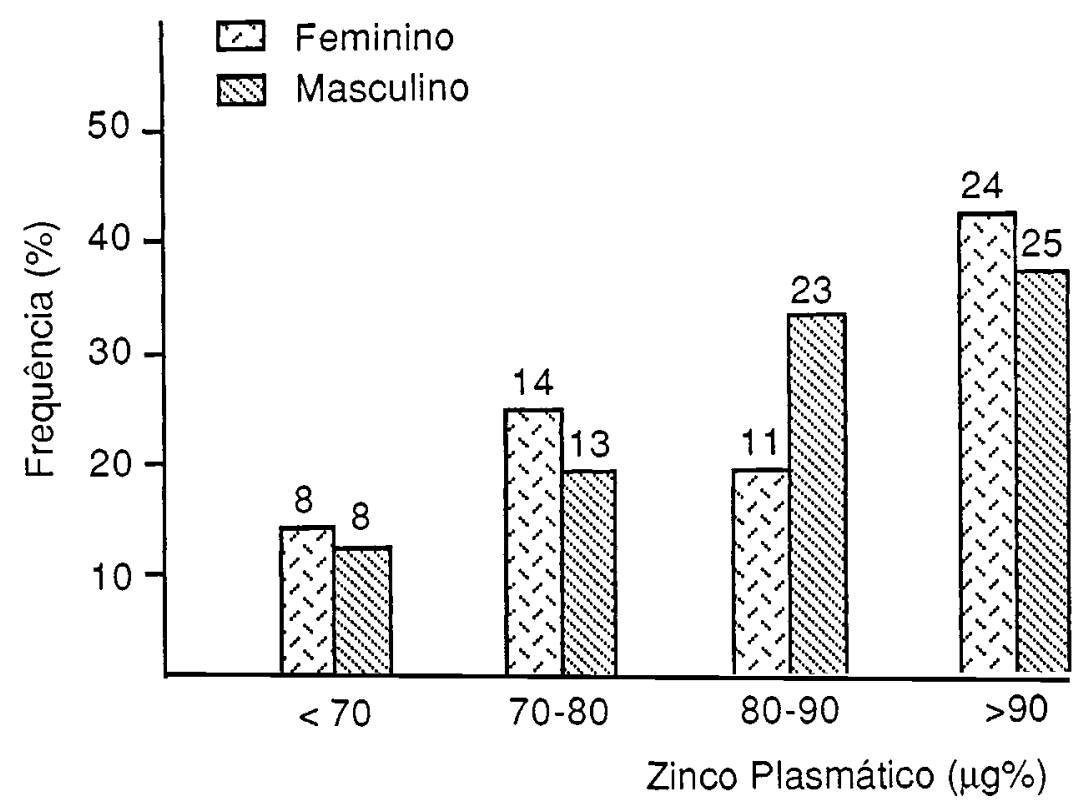

Fig. 2-Distribuição dos níveis plasmáticos de zinco das crianças de famílias de baixa renda de Ribeirão Preto, SP.

acordo com peso, altura, peso/altura, prega tricipital, circunferência braquial e circunferência muscular está apresentada na Figura 1. Das crianças estudadas, $42,9 \%$ apresentaram peso inferior a $90 \%$ do percentil 50 do NCHS. O parâmetro altura por idade de $6,3 \%$ das crianças apresentou valor inferior a $90 \%$ do percentil 50 do NCHS. Encontraram-se, nesta casuística, valores inferiores a $90 \%$ do percentil 50 do NCHS dos seguintes parâmetros: peso/altura $(9,5 \%)$, prega tricipital $(65,8 \%)$, circunferência braquial (11\%) e circunferência mus- cular $(7,3 \%$ das crianças).

\section{Níveis Plasmáticos de Zinco}

A freqüência da distribuição dos níveis plasmáticos de zinco está apresentada na Figura 2. Das crianças estudadas, $14 \%$ das meninas e $11,7 \%$ dos meninos apresentaram niveis plasmáticos de zinco inferiores a $70 \mu \mathrm{g} \% ; 24,6 \%$ das meninas e $18,8 \%$ dos meninos, entre 70 e $79,9 \mu \mathrm{g} \% ; 19,3 \%$ das meninas e $33,3 \%$ dos meninos, entre 80 e $89,9 \mu \mathrm{g} \%$; e $42,1 \%$ das

\section{TABELA 1}

Concentrações plasmáticas de zinco de crianças de famnlias de baixa renda, de acordo com sexo e idade.

\begin{tabular}{|c|c|c|c|c|c|c|}
\hline \multirow{3}{*}{$\begin{array}{l}\text { Idade } \\
\text { (anos) }\end{array}$} & \multicolumn{5}{|c|}{ Concentração plasmática de zinco $(\mu \mathrm{g} / 100 \mathrm{ml})$} & \\
\hline & \multicolumn{2}{|r|}{ Feminino } & \multicolumn{2}{|c|}{ Masculino } & \multicolumn{2}{|c|}{ Ambos } \\
\hline & $\mathrm{n}$ & Média \pm DP & $\mathrm{n}$ & Média $\pm \mathrm{DP}$ & $\mathrm{n}$ & Média $=\mathrm{DP}$ \\
\hline $2-2,9$ & 9 & $86,77 \pm 10,39$ & 7 & $90,51 \pm 21,89$ & 16 & $88,41 \pm 15,91$ \\
\hline $3-3,9$ & 7 & $91,16 \pm 19,94$ & 12 & $93,59 \pm 16,22$ & 19 & $92,69 \pm 17,17$ \\
\hline $4-4,9$ & 13 & $92,15 \pm 14,54$ & 13 & $91,12 \pm 7,71$ & 26 & $91,64 \pm 11,41$ \\
\hline $5-5,9$ & 19 & $85,92 \pm 19,79$ & 27 & $85,19 \pm 14,27$ & 46 & $85,49 \pm 16,57$ \\
\hline $6-6,9$ & 9 & $91,08 \pm 14,97$ & 10 & $81,54 \pm 13,65$ & 19 & $86,06 \pm 14,72$ \\
\hline
\end{tabular}

Diferença entre idade e sexo não significante estatisticamente. $\mathbf{n}=$ tamanho da amostra $\mathrm{DP}=$ Desvio Padrão 


\section{TABELA 2}

Correlação entre níveis plasmáticos de zinco e parâmetros antropométricos de crianças de baixa renda

\begin{tabular}{lccc}
\hline $\begin{array}{c}\text { Parâmetro } \\
\text { antropométrió } \\
\text { (\% do padrăo) }\end{array}$ & $\mathbf{n}$ & $\mathbf{r}$ & $\mathbf{P}$ \\
\hline Peso & 126 & $-0,1749$ & NS \\
Altura & 126 & $-0,0106$ & NS \\
Peso/altura & 126 & $-0,1586$ & NS \\
Prega tricipital & 82 & 0,0593 & NS \\
Circunderência Braquial & 82 & $-0,0345$ & NS \\
Circuferência Muscular & 82 & $-0,1727$ & NS \\
\hline
\end{tabular}

$\mathrm{n}=$ tamanho da amostra

$r=$ coeficiente de correlação

$P=$ Probabilidade

NS = Não significante

meninas e $36,2 \%$ dos meninos apresentaram níveis superiores a $90 \mu \mathrm{g} \%$. A Tabela 1 mostra a média e desvio padrão das concentrações plasmáticas de acordo com o sexo e idade. A análise de variância não mostrou diferenças estatisticamente significantes segundo idade e sexo.

\section{Correlações entre Níveis Plasmáticos de Zinco e Parâmetros Antropométricos}

Os coeficientes de correlação entre níveis plasmáticos de zinco e os parâmetros: peso, altura, peso/altura, prega tricipital, circunferência braquial e muscular de todas as crianças estudadas não são estatisticamente significantes, como mostra a Tabela 2.

\section{DISCUSSĀO}

Das 126 crianças estudadas nesse trabalho, $13 \%$ apresentou níveis plasmáticos de zinco considerados deficientes.

Para caracterizar o estado nutricional, em relação ao zinco, tem-se medido esse mineral nos fluidos biológicos e em vários tecidos, como: plasma, soro, saliva, suor, hemácias, glóbulos brancos, pele, cabelo, unha e urina. O parâmetro mais freqüentemente utilizado tem sido a medida do zinco plasmático ou sérico por meio de espectrofotometria de absorção atômica ${ }^{30}$, apesar dos cuidados necessários para a interpretaçăo dos resultados, uma vez que algumas situações, tais como estresse e infecções, provocam uma redução desses valores ${ }^{4}$.

A deficiência desse mineral tem sido observada em outras regiōes brasileiras. Assim, em trabalhadores de baixa renda de Manaus, AM, mos- tram-se baixos os níveis plasmáticos de zinco ${ }^{27}$. Em crianças, Rocha e col. ${ }^{26}$, em Manaus, e Donangelo e Azevedo ${ }^{10}$, no Rio de Janeiro, encontraram também valores deficientes, especialmente naquelas com processo de desnutrição protéico-calórica. Usando a dosagem do zinco no cabelo também tem-se chamado a atenção para a existência de uma inadequação desse nutriente em crianças brasileiras ${ }^{9,11,12}$.

De forma semelhante a outros trabalhos, nāo se observam, neste estudo, diferenças significativas entre os valores plasmáticos de zinco e o sexo e ainda entre as diferentes faixas de idade ${ }^{14}$.

As observaçōes de deficiência de zinco em adolescentes com nanismo nutricional vem motivando estudos para avaliação da influência do estado nutricional em relação ao nutriente no crescimento ${ }^{25}$. Estudos em animais de laboratório demonstram que o oferecimento de dieta deficiente leva ao retardo de crescimento, com menor síntese muscular ${ }^{15}$. Os valores de zinco, no cabelo, apresentaram correlação positiva com o parâmetro peso/ altura em um estudo realizado na China ${ }^{33}$, o mesmo nảo acontecendo com crianças estudadas em Ilhéus, $\mathrm{BA}^{12}$ e Planaltina, GO $\mathrm{G}^{11}$. Chiou e col. ${ }^{8}$ encontraram uma correlação positiva entre os valores de zinco plasmáticos e área muscular do braço e, por outro lado, correlação negativa com os dados de prega tricipital e área gordurosa do braço. No presente estudo, porém, não se observou correlação significativa entre os níveis plasmáticos de zinco e os dados antropométricos de: peso, altura, relaçăo peso/altura, prega tricipital, circunferência braquial e circunferência muscular.

A suplementaçăo de zinco fornecida a crianças deficientes, comparadas às crianças recebendo placebo mostram, do mesmo modo, resultados diversos, ora com maior ganho de peso $^{32}$, ora com maior velocidade de crescimento na altura ${ }^{16,18}, \mathrm{e}$, ao mesmo tempo, sem diferenças no crescimento do grupo suplementado ${ }^{5,28}$.

Desta forma, os dados existentes parecem ser ainda insuficientes para a conclusão definitiva sobre o papel exato da deficiência de zinco no crescimento e desenvolvimento corporal ao nivel populacional. Outro aspecto, também importante, é a impossibilidade de afastar a existência de deficiências simultâneas de outros nutrientes, que poderiam estar concorrendo para uma alteração no crescimento.

Conclui-se dos dados do presente estudo que, devido aos baixos níveis plasmáticos de zinco encontrados, a ingestão dietética desse nutriente pode ser insuficiente entre crianças de famílias de baixa renda em Ribeirão Preto, SP, entretanto sem repercussão evidente sobre o seu desenvolvimento pondero-estatural. 
FÁVARO, R.M.D. \& VANNUCCHI, H. [Plasma zinc levels and the anthropometry of children on the outskirts of the urban center, Brazill. Rev. Saúde públ., S. Paulo, 24:5 - 10, 1990.

ABSTRACT: The main objective of the present study was to estimate plasma zinc concentration in children belonging to low-income families residing in three different neighborhoods on the outskirts of Ribeiräo Preto, SP, Brazil, and to determine a possible correlation of this parameter with several anthropometric measurements. Plasma zinc levels were lower than $70 \mu \mathrm{g} \%$ in $13 \%$ of the children studied. Weight for age, height for age and weight/height ratio were below $90 \%$ of the 50th percentile of $\mathrm{Na}$ tional Center for Health Statistics (NCHS) in $42.9 \%, 6.3 \%$ and $9.5 \%$ of children, fespectively. Tricipital fold, arm circumference and muscle circumference were also below $90 \%$ of the $50^{\text {th }}$ percentile in $65.8 \%$, $11.0 \%$ and $7.3 \%$ of the children, respectively. No correlation was observed between plasma zinc levels and the anthropometric parameters studied.

KEYWORDS: Zinc, blood. Anthropometry. Socioeconomic factors.

\section{REFERÊNCIAS BIBLIOGRÁFICAS}

1. ABBASI, A. A.; PRASAD, A. S.; RABBANI, P.; DU MOUCHELLE, E. Experimental zinc deficiency in man: effect on testicular function. J. Lab. clin. Med., 96: 544-50, 1980.

2. BJORKSTEN, B.; BACK, 0.; GUSTAVSON, K.H.; HALLMANS, G.; HAGGLOE, B.; TARVNIK, A. Zinc and immune function in Down's syndrome. Acta paediatr. scand., 69: 183-7, 1980.

3. BOGDEN, J.D.; OLESKE, J.M.; MUNVES, E.M. Zinc and immunocompetence in the elderly: baseline data on zinc nutriture and immunity in unsupplemented subjects. Amer. J. clin. Nutr., 45: 101-9, 1987.

4. BUZINA, R.; JUSIC, M.; SAPUNAR, J.; MILANOVIĆ, $N$. Zine nutrition and taste acuity in school children with impaired growth. Amer.J. clin. Nutr., 33: 2262-7, 1980.

5. CARTER, J.P.; GRIVETTI, L.E.; DAVIES, J.T. Growth and sexual development of adolescent Egyptian village boys: effects of zinc, iron and placebo supplementation. Amer. J, clin. Nutr., 22: 59-78, 1969.

6. CASTILLO-DURAN, C.; HERESI, G.; FISBERG, M.; UAUY, R. Controlled trial of zinc supplementation during recovery from malnutrition: effects on growth and immune function. Amer. J. clin. Nutr., 45; 602-8, 1987.

7. CHANDRA, R.K. \& KUTTY, K.M. Immunocompetence in obesity. Acta paediatr. scand., 69: 25-30, 1980.

8. CHIOU, C.J.; KO, Y.C.; LN, S.M. A study of the correlation between levels of serum minerals and the physical growth of school-age children on the off-shore Island of Penghy. Kaohsiung J. med. Sci., 2: 664-8, 1986.

9. CTENAS, M.L.B.; AGOSTINI, S.B.; TONDDN, H.E.; MOYSÉS, M.C.J. Concentração de zinco no cabelo de crianças de 5 a 74 meses. In: Congresso Nacional da Sociedade Brasileira de Nutrição e Alimentação, 1², São Paulo, 1987. Programas e resumos. São Paulo, 1987. p. 175.

10. DONANGELO, C.M. \& AZEVEDO, C.E. Zinco sérico em crianças brasileiras de familias de baixa renda. Arch. latinoamer. Nutr., 34: 290-7, 1984.

11. DÓREA, J. G.; ALMEIDA, I. S.; QUEIROZ, E. F.O.; HORNER, M.R. Nutritional status and zinc nutriture in infants and children in a poor urban community of Bra- zil. Ecol. Food Nutr., 12: 1-6, 1982.

12. DÓREA, J.G.; HORNER M.R.; BEZERRA, V.L; PEREIRA, M.G.; SALOMON, J.B. Hair zinc levels and nutritional status in urban children from Ithéus, Bahia, Brazil. Hum. Nutr. appl. Nutr., 36A: 63-7, 1982.

13. FRISANCHO, A.R. New norms for upper limb fat and muscle areas for assessment of nutritional status. Amer. J. clin. Nutr., 34: 2540-5, 1981.

14. GOLDEN, B.L. \& GOLDEN, M.H.N. Plasma zinc and the clinical features of malnutrition. Amer. J. clin. Nutr., 32: 2490-4, 1979.

15. GUIGLIANO, R. \& MILLWARD, D.J. Growth and zinc homeostasis in the severely $\mathrm{Zn}$ deficient rat. Brit. $J$. Nutr., 52: 545-60, 1984.

16. HALSTED, J.A.; RONAGHY, H.A.; ABADI, P.; HAGHSHENASS, M.; AMIRHEKEMI, G.J.; BARAKET, R.N.; RENNHOLD, J.G. Zinc deficiency in man: the Shiraz experiment. Amer. J. Med., 53: 277-83, 1972.

17. HAMBIDGE, K. M.; WALRAVENS, P. A.; BROWN, R.M.; WEBSTER, J.; WHITE, S.; ANTHONY, M.; ROTH, M.L Zinc nutrition of preschool children in the Denver Head Start Program. Amer. J. clin. Nutr., 29: 7348, 1976.

18. HAMBIDGE, K.M. Zinc deficiency in the premature infant. Pediatr. Rev., 6: 209-16, 1985.

19. HAMILL, P. V. V.; DRIZD, T. A.; JOHNSON, C. L.; REED, R.B.; ROCHE, A.F.; MOORE, W.M. Physical growth: National Center for Health Statistic Percentiles. Amer. J. clin. Nutr., 32: 607-29, 1979.

20. JELLIFFE, D.B. The assessment of the nutritional status of the community. Geneva, World Health Organization, 1966.

21. KREBS, N.F.; HAMBIDGE, K.M.; WALRAVENS, P.A. Increased food intake of young children receiving a zinc supplement. Amer. J. Dis. Child., 138: 270-73, 1984.

22. LAMOUNIER, J.A.; DANELLUZZI, J.C.; VANNUCCHI, $\mathrm{H}$. Zinc concentrations in human milk during lactation: a 6-month longitudinal study in Southem Brazil. $J$. trop. Pediat., 35: 31-4, 1989. 
23. MAHAJAN, S. R.; PRASAD, A. S.; RABBANI, P.; BRIGGS, W.A.; McDONALD, F.D. Zinc deficiency: a reversible complication of uremia. Amer. J. clin. Nutr., 36: 1177-83, 1982.

24. MULS, C.F. Zinc in human biology. London, SpringerVerlag, 1988.

25. PRASAD, A.S.; SCHULERT, A.R.; MIALE, A.; FARID, Z.; SANDSTEAD, H.H. Zinc metabolism in patients with the syndrome of iron deficiency anemia, hepatosplenomegaly, dwarfism and hypogonadism. J. Lab. clin. Med., 61: 537-42, 1963.

26. ROCHA, Y.R. da; MARINHO, H.A.; CASTRO, J.S. de; FERRARONI, M.J.R.; NAGAHAMA, D.; SILVA, N. B. da. Níveis de zinco sérico em pré-escolares de baixa renda em Manaus - AM. In: Congresso Nacional da Sociedade Brasileira de Nutrição e Alimentação, $1^{2}$, São Paulo, 1987. Programa e resumos. São Paulo, 1987. p. 173.

27. SHRIMPTON, R.; FRANCA, T. S.; ROCHA, Y.R.; GOLDEN, M.H.N. Estudo sobre o estado nutricional em relação ao zinco na Amazônia. Acta amazôn., 13: 73-94, 1983.

28. SMTTH, R.M.; KING, R.A.; SPARGO, R.M.; CHEEK, D.B.; FIELD, J.B.; VEITCH, L.G. Growth-retarded ab- original children with low plasma zinc levels do not show a growth response to supplementary zinc. Lancet, 1: $923-4,1985$.

29. SNEDECOR, G.W. \& COCHRAN, N.G. Statistical methods. 6th ed. Ames, Ia., Iowa University Press, 1967.

30. SOLOMONS, N.W. Zinc and copper. In: SHILS, M.E. \& YOUNG, V.R. Modern nutrition in health and disease. 7th ed. Philadelphia, Lea \& Febiger, 1988. p. 238-62.

31. VANDERKOOY, P.D.S. \& GIBSON, R.S. Food consumption patterns of Canadian preschool children in relation to zinc and growth status. Amer. J. clin. Nutr., 45: 609-16, 1987.

32. WALRAVENS, P.A. \& HAMBIDGE, K.M. Growth of infants fed a zinc supplemented formula. Amer. J. clin. Nutr., 29: 1114-21, 1976.

33. XUE-CUN, C.; TAI-AN, Y;; YIN-SHENG, He; QIUYAN, M; ZHI-MIN, H.; LI-XIANG, Li. Low levels of zinc in hair and blood, pica, anorexia, and poor growth in chinese preschool children. Amer. J. clin. Nutr., 42: 694$700,1985$.

Recebido para publicaçäo em $21 / 11 / 1988$ Reapresentado em 11/10/1989 Aprovado para publicaçäo em 13/10/1989 